\title{
Optimum Secret Sharing Scheme Secure against Cheating
}

\author{
Wakaha Ogata ${ }^{1}$ and Kaoru Kurosawa ${ }^{2}$ \\ 1 Himeji Institute of Technology, 2167 Shosha, Himeji-shi, Hyogo 671-22, Japan \\ wakaha@comp.eng.himeji-tech.ac.jp \\ 2 Tokyo Institute of 'Technology, 2-12-1 O-okayama, Meguro-ku, Tokyo 152, Japan \\ kurosawa@ss.titech.ac.jp
}

\begin{abstract}
Tompa and Woll considered a problem of cheaters in $(k, n)$ threshold secret sharing schemes. We first derive a tight lower bound on the size of shares $\left|\mathcal{V}_{i}\right|$ for this problem: $\left|\mathcal{V}_{i}\right| \geq(|\mathcal{S}|-1) / \delta+1$, where $\mathcal{V}_{i}$ denotes the set of shares of participant $P_{i}, \mathcal{S}$ denotes the set of secrets, and $\delta$ denotes the cheating probability. We next present an optimum scheme which meets the equality of our bound by using "difference sets."
\end{abstract}

\section{Introduction}

$(k, n)$ threshold secret sharing schemes $[2,3]$ have been studied extensively so far because of their wide applications in fields, like key management and secure computation. In such a scheme, a dealer $D$ distributes a secret $s$ to $n$ participants $P_{1}, \ldots, P_{n}$ in such a way that any $k$ or more participants can recover the secret $s$ but any $k-1$ or fewer participants have no information on $s$. A piece of information given to $P_{i}$ is called a share and is denoted by $v_{i}$. An important issue in sccret sharing schemes is the size of shares $\left|\mathcal{V}_{i}\right|$, where $V_{i} \triangleq\left\{v_{i} \mid \operatorname{Pr}\left(v_{i}\right)>0\right\}$, because the security of a system will decrease if $\left|\mathcal{V}_{i}\right|$ increases. Let $\mathcal{S} \triangleq\{s \mid$ $\operatorname{Pr}(s)>0\}$. Then it is known that

$$
\left|\mathcal{V}_{i}\right| \geq|\mathcal{S}|
$$

in any $(k, n)$ threshold scheme [4].

Tompa and Woll [1] considered the following scenario. Suppose that $k-1$ participants $P_{1}, \ldots, P_{k-1}$ want to cheat a $k$-th participant $P_{k}$ by opening forged shares $v_{1}^{\prime}, \ldots, v_{k-1}^{\prime}$. They succeed if the secret $s^{\prime}$ reconstructed from $v_{1}^{\prime}, \ldots, v_{k-1}^{\prime}$ and $v_{k}$ is different from the original secret $s$. Tompa and Woll showed that Shamir's scheme [2] is insecure against this attack in that even a single participant can, with high probability, deceive $k-1$ honest participants. They showed a scheme secure against this problem, but $\left|\mathcal{V}_{i}\right|$ in their scheme is very large:

$$
\left|\mathcal{V}_{i}\right|=((|\mathcal{S}|-1)(k-1) / \epsilon+k)^{2}
$$

where $\epsilon$ denotes the cheating probability. Carpentieri, De Santis, and Vaccaro [5] recently showed the following lower bound on $\left|\mathcal{V}_{i}\right|$ for this problem:

$$
\left|\mathcal{V}_{i}\right| \geq|\mathcal{S}| / \epsilon
$$


Now, we see that there is a big gap between eq. (1) and (2). Both of them can be improved. Furthermore, in the derivation of eq. (2) it is assumed that $k-1$ cheaters $P_{1}, \ldots, P_{k-1}$ somehow know the secret $s$ before they cheat $P_{k}$. (We call this the CIDV assumption.)

In this paper we first derive a tight lower bound on $\left|\nu_{i}\right|$ for this problem by using a probabilistic method. In deriving our bound, we do not use the CDV assumption. That is, it is assumed that $k-1$ cheaters have no information on $s$ (according to the definition of $(k, n)$ threshold secret sharing schemes). Let $\delta$ be the probability that $P_{1}, \ldots, P_{k-1}$ can cheat $P_{k}$. Then our bound is

$$
\left|\mathcal{V}_{i}\right| \geq(|\mathcal{S}|-1) / \delta+1
$$

We then present an optimum scheme which meets the equality of our bound by using "difference sets." A planar difference set modulo $N=l(l-1)+1$ is a set of $l$ numbers $B=\left\{d_{0}, d_{1}, \ldots, d_{l-1}\right\}$ with the property that the $l(l-1)$ differences $d_{i}-d_{j}\left(d_{i} \neq d_{j}\right)$, when reduced modulo $N$, are exactly the numbers $1,2, \ldots, N-1$ in some order [6]. It is known that there exists a planar difference set if $l$ is a prime power [6]. Our optimum scheme is then characterized as follows. If there exists a planar difference set modulo $N=l(l-1)+1$ such that $N$ is a prime, then there exists a $(k, n)$ threshold secret sharing scheme which meets the equality of our bound eq. (3) such that $|\mathcal{S}|=l, \delta=1 / l, n<N$.

Furthermore, this result is generalized as follows. Let $(\Gamma,+)$ be a group of order $N$ and let $B=\left\{d_{0}, d_{1}, \ldots, d_{-1}\right\}$ be a subset of $\Gamma$. Then $B$ is called a $(N, l, \lambda)$ difference set [7] if each nonzero element $x$ of $\Gamma$ appears $\lambda$ times as a difference $d_{i}-d_{j}\left(d_{i} \neq d_{j}\right)$. Our generalized scheme is then given as follows. There exists a $(k, n)$ threshold secret sharing scheme which meets the equality of our bound eq. (3) such that $|\mathcal{S}|=l, \delta=\lambda / l, n<N$ if there exists a $(N, l, \lambda)$ difference set $B$ in $(G F(N),+)$. It is known that there exists a $(N, l, \lambda)$ difference set $B$ in $(G F(N),+)$ such that $N=4 t-1, l=2 t-1, \lambda=t-1[7]$.

Finally, for the model with the CDV assumption, we show a lower bound on $\left|\mathcal{V}_{i}\right|$ more tight than eq. (2) by using the same technique we use to derive eq. (3). Our bound for the model with the CDV assumption is

$$
\left|\mathcal{V}_{i}\right| \geq(|\mathcal{S}|-1) / \epsilon^{2}+1
$$

A slightly different problem has been studied by other researchers. McElice and Sarwate [8] showed that in Shamir's $(k, n)$ threshold scheme, any group of $k+$ $2 e$ participants which includes at most $e$ cheaters can always identify cheaters and correctly calculate the secret. (More than $k$ participants are required though.) The problem of identifying cheaters has also been studied $[9,10,11,12]$. Those schemes, however, require $\left|\mathcal{V}_{i}\right|$ much bigger than the bound given in eq. (3). On the other hand, in this paper, we are interested only in detecting the fact of cheating. 


\section{Preliminaries}

\subsection{Definition of cheating}

$D$ denotes a probabilistic Turing machine called a dealer, $S$ denotes a random variable distributed over a finite set $\mathcal{S}$, and $s \in \mathcal{S}$ is called a secret. On input $s \in \mathcal{S}, D$ outputs $\left(v_{1}, \ldots, v_{n}\right)$ randomly. For $1 \leq i \leq n$, each participant $P_{i}$ holds $v_{i}$ as his share. $V_{i}$ denotes the random variable induced by $v_{i}$. Let $\mathcal{V}_{i} \triangleq\left\{v_{i} \mid\right.$ $\left.\operatorname{Pr}\left(V_{i}=v_{i}\right)>0\right\}$.

Definition 1. We say that $(D, S)$ is a $(k, n)$ threshold secret sharing scheme if the following two requirements hold: For any $\left\{i_{1}, \ldots, i_{j}\right\} \subseteq\{1, \ldots, n\}$ and $\left(v_{i_{1}}, \ldots, v_{i_{j}}\right)$ such that $\operatorname{Pr}\left(V_{i_{1}}=v_{i_{1}}, \ldots, V_{i_{j}}=v_{i_{j}}\right)>0$,

(A1) if $j \geq k$, there exists a unique $s \in \mathcal{S}$ such that

$$
\operatorname{Pr}\left(S=s \mid V_{i_{1}}=v_{i_{1}}, \ldots, V_{i_{j}}=v_{i_{j}}\right)=1,
$$

(A2) if $j<k$, for each $s \in \mathcal{S}$,

$$
\operatorname{Pr}\left(S=s \mid V_{i_{1}}=v_{i_{1}}, \ldots, V_{i_{j}}=v_{i_{j}}\right)=\operatorname{Pr}(S=s) .
$$

Definition 2. For $w \in \mathcal{V}_{i_{1}} \times \cdots \times \mathcal{V}_{i_{k}}$,

$$
\operatorname{Sec}_{\left(i_{1}, \ldots, i_{k}\right)}(w) \triangleq \begin{cases}s & \text { if } \exists s \in S \text { such that } \operatorname{Pr}\left(S=s \mid V_{i_{1}} \cdots V_{i_{k}}=w\right)=1, \\ \perp & \text { otherwise. }\end{cases}
$$

$\left(\left(i_{1}, \ldots, i_{k}\right)\right.$ will be omitted. $)$

Definition 3. Suppose that $k-1$ cheaters $P_{i_{1}}, \ldots, P_{i_{k-1}}$ have $b=\left(v_{i_{1}}, \ldots, v_{i_{k-1}}\right)$ as their shares. We say that the cheaters can cheat $P_{i_{k}}$ by opening $b^{\prime}=\left(v_{i_{1}}^{\prime}, \ldots, v_{i_{k-1}}^{\prime}\right)$ if $\operatorname{Sec}\left(b^{\prime}, v_{i_{k}}\right) \neq \operatorname{Sec}\left(b, v_{i_{k}}\right)$ and $\operatorname{Sec}\left(b^{\prime}, v_{i_{k}}\right) \in \mathcal{S}$, where $v_{i_{k}}$ denotes the share of $P_{i_{k}}$.

\subsection{Known bound on $\left|\mathcal{V}_{i}\right|$ under the CDV assumption}

Carpentieri, De Santis, and Vaccaro [5] showed the following lower bound on $\left|V_{i}\right|$ by using entropy. In deriving that bound they assumed that $k-1$ cheaters $P_{i_{1}}, \ldots, P_{i_{k-1}}$ shomehow know the secret $s$ before they cheat $P_{k}$, although, in the definition of $(k, n)$ threshold secret sharing schemes, $k-1$ cheaters have no information on $s$. (We call this the CDV assumption.) Let $b=\left(v_{i_{1}}, \ldots, v_{i_{k-1}}\right)$ denote the shares of the cheaters, and let $b^{\prime}=\left(v_{i_{1}}^{\prime}, \ldots, v_{i_{k-1}}^{\prime}\right)$ denote the forged shares that the cheaters open to cheat $P_{i_{k}}$. Carpentieri et al. defined the average cheating probability as follows:

$$
\begin{aligned}
P^{\prime}\left(\text { Cheat } \mid V_{i_{1}}, \ldots, V_{i_{k-1}}, S\right) \triangleq & E\left[\operatorname { m a x } _ { b ^ { \prime } } \operatorname { P r } \left(P_{i_{k}} \text { is cheated by } b^{\prime}\right.\right. \\
& \left.\left.\mid P_{i_{1}} \ldots P_{i_{k-1}} \text { have } b \text {. They also know } s\right)\right],
\end{aligned}
$$


Definition 4. [5] A $(k, n)$ threshold secret sharing scheme is called a $(k, n, \epsilon)$ robust secret sharing scheme if $P^{\prime}\left(C\right.$ heat $\left.\mid V_{i_{1}}, \ldots, V_{i_{k-1}}, S\right) \leq \epsilon$ for any $\left\{i_{1}, \ldots, i_{k-1}\right\} \subseteq\{1, \ldots, n\}$.

Proposition 5. [5] In a $(k, n, \epsilon)$ robust secret sharing scheme, if the secret is uniformly chosen, then $\left|\mathcal{V}_{i}\right| \geq|\mathcal{S}| / \epsilon$.

\section{New Lower Bound on $\left|\mathcal{V}_{i}\right|$}

\subsection{Definition of secure secret sharing}

In this section we derive a tight lower bound on $\left|\mathcal{V}_{i}\right|$ by using a probabilistic method. In deriving this bound we do not make the CDV assumption (see subsection 2.2). That is, it is assumed that, according to the definition of $(k, n)$ threshold secret sharing schemes, $k-1$ cheaters have no information on $s$. Suppose that $P_{i_{1}}, \ldots, P_{i_{k-1}}$ are cheaters. Let $b=\left(v_{i_{1}}, \ldots, v_{i_{k-1}}\right)$ denote the shares of the cheaters, and let $b^{\prime}=\left(v_{i_{1}}^{\prime}, \ldots, v_{i_{k-1}}^{\prime}\right)$ denote the forged shares that the cheaters open to cheat $P_{i_{k}}$. Since the cheaters have no information on $s$, we define the average cheating probability as follows:

$$
\begin{aligned}
& P\left(C h c a t \mid V_{i_{1}}, \ldots, V_{i_{k-1}}\right) \\
& \triangleq E\left[\max _{b^{\prime}} \operatorname{Pr}\left(P_{i_{k}} \text { is cheated by } b^{\prime} \mid P_{i_{1}} \cdots P_{i_{k-1}} \text { have } b\right)\right]
\end{aligned}
$$

( $S$ and $s$ in eq. (4) are absent from eq. (5).)

Definition 6. A $(k, n)$ threshold secret sharing scheme is called a $(k, n, \delta)$ secure secret sharing scheme if $P\left(\right.$ Cheat $\left.\mid V_{i_{1}}, \ldots, V_{i_{k-1}}\right) \leq \delta$ for any $\left\{i_{1}, \ldots, i_{k-1}\right\} \subseteq$ $\{1, \ldots, n\}$.

\subsection{New lower bound on $\left|\mathcal{V}_{i}\right|$}

In the distribution phase, suppose that cheaters $P_{i_{1}}, \ldots, P_{i_{k-1}}$ have $b=\left(v_{i_{1}}, \ldots, v_{i_{k-1}}\right)$ as their shares of a secret $s$ and $P_{i_{k}}$ has $x$ as his share. That is, $\operatorname{Sec}(b, x)=s$. In the reconstruction phase, if $P_{i_{1}}$ opens $v_{i_{1}}^{\prime}\left(\neq v_{i_{1}}\right)$ such that $\operatorname{Sec}\left(v_{i_{1}}^{\prime}, v_{i_{2}}, \ldots, v_{i_{k-1}}, x\right)=$ $s^{\prime}$ and $s^{\prime} \neq s$, then $P_{i_{k}}$ is cheated. Now, let

$$
Y(x, s) \triangleq\left\{v_{i_{1}}^{\prime} \in \mathcal{V}_{i_{1}} \mid \operatorname{Sec}\left(v_{i_{1}}^{\prime}, v_{i_{2}}, \ldots, v_{i_{k-1}}, x\right)=s^{\prime} \in \mathcal{S}, s^{\prime} \neq s\right\}
$$

For fixed $x$ and $s, Y(x, s)$ denotes the set of forged shares of $P_{i_{1}}$ which can cheat $P_{i_{k}}$. (However, the cheaters do not know $x$ nor s.) Let

$$
W(s) \triangleq\left\{x \in \mathcal{V}_{i_{k}} \mid \operatorname{Sec}(b, x)=s\right\} .
$$

$W(s)$ denotes the set of possible shares of $P_{i_{k}}$ for a fixed $s$.

Lemma 7. For $\forall s \in \mathcal{S}, \forall x \in W(s)$,

$$
|Y(x, s)| \geq|\mathcal{S}|-1 \text {. }
$$


Proof. Since $k$ participants can recover the secret uniquely, for $\forall s^{\prime}, s^{\prime \prime}\left(s^{\prime} \neq s^{\prime \prime}\right)$,

$$
\begin{aligned}
& \left\{v_{i_{1}}^{\prime} \in \mathcal{V}_{i_{1}} \mid \operatorname{Sec}\left(v_{i_{1}}^{\prime}, v_{i_{2}}, \ldots, v_{i_{k-1}}, x\right)=s^{\prime}\right\} \\
& \cap\left\{v_{i_{1}}^{\prime} \in \mathcal{V}_{i_{1}} \mid \operatorname{Sec}\left(v_{i_{1}}^{\prime}, v_{i_{2}}, \ldots, v_{i_{k-1}}, x\right)=s^{\prime \prime}\right\}=\emptyset
\end{aligned}
$$

From (A2) of Def.1, for any $s^{\prime} \in \mathcal{S}$, there exists at least one $v_{k_{1}}^{\prime}$ such that

$$
\operatorname{Sec}\left(v_{i_{1}}^{\prime}, v_{i_{2}}, \ldots, v_{i_{k-1}}, x\right)=s^{\prime} .
$$

Therefore, from the definition of $Y(x, s)$,

$$
\begin{aligned}
|Y(x, s)| & =\left|\bigcup_{s^{\prime} \in \mathcal{S}, s^{\prime} \neq s}\left\{v_{i_{1}}^{\prime} \in \mathcal{V}_{i_{1}} \mid \operatorname{Sec}\left(v_{i_{1}}^{\prime}, v_{i_{2}}, \ldots, v_{i_{k-1}}, x\right)=s^{\prime}\right\}\right| \\
& =\sum_{s^{\prime} \in \mathcal{S}, s^{\prime} \neq s}\left|\left\{v_{i_{1}}^{\prime} \in \mathcal{V}_{i_{1}} \mid \operatorname{Sec}\left(v_{i_{1}}^{\prime}, v_{i_{2}}, \ldots, v_{i_{k-1}}, x\right)=s^{\prime}\right\}\right| \\
& \geq \sum_{s^{\prime} \in \mathcal{S}, s^{\prime} \neq s} 1 \\
& =|\mathcal{S}|-1 .
\end{aligned}
$$

Now our lower bound on $\left|\mathcal{V}_{i}\right|$ is given as follows. The following bound holds for any distribution on $S$.

Theorem 8. In a $(k, n, \delta)$ secure secret sharing scheme,

$$
\left|\mathcal{V}_{i}\right| \geq \frac{|\mathcal{S}|-1}{\delta}+1
$$

Proof. Consider cheaters $P_{i_{1}}, \ldots, P_{i_{k-1}}$ such that only $P_{i_{1}}$ opens a forged share $v_{i_{1}}^{\prime}\left(\neq v_{i_{1}}\right)$. The other $P_{i_{2}}, \ldots, P_{i_{k-1}}$ open their shares honestly. For these specific cheaters,

$$
\begin{aligned}
& \max _{b^{\prime}} \operatorname{Pr}\left(P_{i_{k}} \text { is cheated by } b^{\prime} \mid P_{i_{1}} \cdots P_{i_{k-1}} \text { have } b\right) \\
\geq & \max _{v_{i_{1}}^{\prime}} \operatorname{Pr}\left(P_{i_{k}} \text { is cheated by } v_{i_{1}}^{\prime} \mid P_{i_{1}} \cdots P_{i_{k-1}} \text { have } b\right)
\end{aligned}
$$

Now, we randomize $v_{i_{1}}^{\prime}$ in order to compute the right-hand side. Consider $P_{i_{1}}$ who opens $v_{i_{1}}^{\prime}\left(\neq v_{i_{1}}\right)$ randomly. More precisely,

$$
\operatorname{Pr}\left(P_{i_{1}} \text { opens } v_{i_{1}}^{\prime}\right)= \begin{cases}1 /\left(\left|V_{i_{1}}\right|-1\right) & \text { if } v_{i_{1}}^{\prime} \neq v_{i_{1}} \\ 0 & \text { if } v_{i_{1}}^{\prime}=v_{i_{1}}\end{cases}
$$

For this probabilistic $P_{i_{1}}$, let's compute

$$
E\left[\operatorname{Pr}\left(P_{i_{k}} \text { is cheated by } v_{i_{1}}^{\prime} \mid P_{i_{1}} \cdots P_{i_{k-1}} \text { have } b\right)\right],
$$


where $E$ is taken over $v_{i_{1}}^{\prime}$ and $\operatorname{Pr}()$ is taken over $s$ and $x$. Then from lemma 7 ,

$$
\begin{aligned}
& E_{v_{i_{1}}^{\prime}}\left[\operatorname{Pr}_{s, x \in W(s)}\left(P_{i_{k}} \text { is cheated by } v_{i_{1}}^{\prime} \mid P_{i_{1}} \cdots P_{i_{k-1}} \text { have } b\right)\right] \\
& \left.=E_{s, x \in W(s)} \underset{v_{i_{1}}^{\prime}}{\operatorname{Pr}}\left(P_{i_{k}} \text { is cheated by } v_{i_{1}}^{\prime} \mid P_{i_{1}} \cdots P_{i_{k-1}} \text { have } b\right)\right] \\
& =E_{s, x \in W(s)}\left[|Y(x, s)| /\left(\left|\mathcal{V}_{i_{1}}\right|-1\right)\right] \\
& \geq(|\mathcal{S}|-1) /\left(\left|\mathcal{V}_{i_{1}}\right|-1\right) \text {. }
\end{aligned}
$$

Therefore

$$
\begin{aligned}
& \max _{v_{i_{1}}^{\prime}} \operatorname{Pr}\left(P_{i_{k}} \text { is cheated by } v_{i_{1}}^{\prime} \mid P_{i_{1}} \cdots P_{i_{k-1}} \text { have } b\right) \\
\geq & E_{v_{i_{1}}^{\prime}}\left[\operatorname{Pr}\left(P_{i_{k}} \text { is cheated by } v_{i_{1}}^{\prime} \mid P_{i_{1}} \cdots P_{i_{k-1}} \text { have } b\right)\right] \\
\geq & (|\mathcal{S}|-1) /\left(\left|\mathcal{V}_{i_{1}}\right|-1\right) .
\end{aligned}
$$

Hence, from eq. (7),

$$
\begin{gathered}
\max _{b^{\prime}} \operatorname{Pr}\left(P_{i_{k}} \text { is cheated by } b^{\prime} \mid P_{i_{1}} \cdots P_{i_{k-1}} \text { have } b\right) \geq(|\mathcal{S}|-1) /\left(\left|\mathcal{V}_{i_{1}}\right|-1\right) . \\
E_{b}\left[\max _{b^{\prime}} \operatorname{Pr}\left(P_{i_{k}} \text { is cheated by } b^{\prime} \mid P_{i_{1}} \cdots P_{i_{k-1}} \text { have } b\right)\right] \geq(|\mathcal{S}|-1) /\left(\left|\mathcal{V}_{i_{1}}\right|-1\right) .
\end{gathered}
$$

Consequently, in a $(k, n, \delta)$ secure secret sharing scheme,

$$
\delta \geq P\left(\text { Cheat } \mid V_{i_{1}}, \ldots, V_{i_{k-1}}\right) \geq(|\mathcal{S}|-1) /\left(\left|V_{i_{1}}\right|-1\right)
$$

Therefore, $\left|\mathcal{V}_{i,}\right| \geq(|\mathcal{S}|-1) / \delta+1$.

\section{Optimum $(k, n, \delta)$ Secure Scheme}

In this section, we show an optimum scheme which meets the equality of Theorem 8 by using "difference sets."

\subsection{Difference set}

Definition 9. [6] A planar difference set modulo $N=l(l-1)+1$ is a set of $l$ numbers $B=\left\{d_{0}, d_{1}, \ldots, d_{l-1}\right\}$ with the property that the $l(l-1)$ differences $d_{i}-d_{j}\left(d_{i} \neq d_{j}\right)$, when reduced modulo $N$, are exactly the numbers $1,2, \ldots, N-1$ in some order.

Example 1. [6] $\left\{d_{0}=0, d_{1}=1, d_{2}=3\right\}$ is a planar difference set modulo 7 with $l=3$. Indeed, the differences modulo 7 are

$$
1-0=1,3-0=3,3-1=2,0-1=6,0-3=4,1-3=5 .
$$

Proposition 10. [6] In a projective plane $P G(2, q)$, a line has $l=q+1$ points $\alpha^{d_{0}}, \ldots, \alpha^{d_{l-1}}$, where $q$ is a prime power. Then $\left\{d_{0}, \ldots, d_{l-1}\right\}$ is a planar difference set modulo $q^{2}+q+1$. 
Definition 9 is generalized as follows.

Definition 11. [7] Let $(\Gamma,+)$ be a group of order $N . B$ is called a $(N, l, \lambda)$ difference set if it satisfies

$-B \subset \Gamma$ and $|B|=l$,

- the list of differences $d-d^{\prime} \neq 0$, where $d, d^{\prime} \in B$, contains each nonzero element of $\Gamma$ precisely $\lambda$ times.

Proposition 12. [ 7$]$ There exists a $(N, l, \lambda)$ difference set $B$ in $(G F(N),+)$ such that $N=4 t-1, l=2 t-1, \lambda=t-1$, where $t$ is a positive integer.

Example 2. [7] $B=\{1,3,4,5,9\}$ is a $(11,5,2)$-difference set in $(G F(11),+)$.

\subsection{Optimum scheme based on planar difference set}

In this subsection we show that if there exists a planar difference set modulo $N=l(l-1)+1$ such that $N$ is a prime, then there exists a $(k, n, \delta)$ secure secret sharing scheme which meets the equality of our bound eq. (6) such that $|\mathcal{S}|=l, \delta=1 / l, n<N$.

Let $B=\left\{d_{0}, \ldots, d_{l-1}\right\}$ be a planar difference set modulo $N=l(l-1)+1$ such that $N$ is a prime. We show a $(k, n, \delta)$ secure secret sharing scheme such that $\mathcal{S}=B$. Assume that $S$ is uniformly distributed over $\mathcal{S}$. In what follows, all operations are done over $G F(N)$.

Distribution phase. For a secret $d_{s} \in \mathcal{S}(=B)$, the dealer $D$ chooses a random polynomial $f(x)$ of degree $k-1$ over $G F(N)$ such that $f(0)=d_{s}$. The share of $P_{i}$ is given as $v_{i}=f(i)$. Note that

$$
\forall i, \quad\left|\mathcal{V}_{i}\right|=N=l(l-1)+1
$$

Reconstruction phase. Suppose that $P_{i_{1}}, \ldots, P_{i_{k}}$ open $\tilde{v}_{i_{1}}, \ldots, \tilde{v}_{i_{k}}$. Each participant computes $\tilde{d}_{s}=\sum_{j=1}^{k} c_{j} \tilde{v}_{i_{j}}$, where $c_{j}=\prod_{i \neq j}\left(-i_{l}\right) /\left(i_{j}-i_{l}\right)$ for $1 \leq j \leq k$. If $\tilde{d}_{s} \in B$, they accept $\tilde{d}_{s}$ as the secret. Otherwise, they output $\perp$. Note that, for any $k$ honest shares $v_{i_{1}}=f\left(i_{1}\right), \ldots, v_{i_{k}}=f\left(i_{k}\right)$,

$$
d_{s}=\sum_{j=1}^{k} c_{j} v_{i_{j}}
$$

from Laglange formula [13].

Proposition 13 (Lagrange formula). [19] Let $h(x)$ be a polynomial over $G F(N)$ such that $\operatorname{deg} h(x)=k-1$. For any distinct $i_{1}, \ldots, i_{k}$,

$$
h(0)=\sum_{j=1}^{k} c_{j} h\left(i_{j}\right), \text { where } c_{j}=\prod_{l \neq j}\left(-i_{l}\right) /\left(i_{j}-i_{l}\right)
$$

Lemma 14. The proposed scheme is a $(k, n)$ threshold secret sharing scheme. 
Proof. (A1) of Defnition 1 is satisfied from eq. (9). Next,

$$
\begin{aligned}
& \operatorname{Pr}\left(S=d_{s} \mid V_{i_{1}}=v_{i_{1}}, \ldots, V_{i_{k-1}}=v_{i_{k-1}}\right) \\
& =\frac{\operatorname{Pr}\left(S=d_{s}\right) \operatorname{Pr}\left(V_{i_{1}}=v_{i_{1}}, \ldots, V_{i_{k-1}}=v_{i_{k-1}} \mid S=d_{s}\right)}{\operatorname{Pr}\left(V_{i_{1}}=v_{i_{1}}, \ldots, V_{i_{k-1}}=v_{i_{k-1}}\right)} .
\end{aligned}
$$

For each $d_{s} \in \mathcal{S}, f(x)$ is randomly chosen and $\operatorname{deg} f(x)=k-1$. Therefore $V_{i_{1}} \cdots V_{i_{k-1}}$ is random for each $d_{s} \in \mathcal{S}$. Hence

$$
\operatorname{Pr}\left(V_{i_{1}}=v_{i_{1}}, \ldots, V_{i_{k-1}}=v_{i_{k-1}} \mid S=d_{s}\right)=\operatorname{Pr}\left(V_{i_{1}}=v_{i_{1}}, \ldots, V_{i_{k-1}}=v_{i_{k-1}}\right) .
$$

Consequently,

$$
\operatorname{Pr}\left(S=d_{s} \mid V_{i_{1}}=v_{i_{1}}, \ldots, V_{i_{k-1}}=v_{i_{k-1}}\right)=\operatorname{Pr}\left(S=d_{s}\right) .
$$

Thus (A2) of Defnition 1 is also satisfied.

Lemma 15. The proposed scheme is a $(k, n, \delta)$ secure secret sharing scheme such that $|\mathcal{S}|=l, \delta=1 / l$ and $n<N$. Furthermore, the equality of eq. (6) is satisfied.

Proof. Suppose that cheaters $P_{i_{1}}, \ldots, P_{i_{k-1}}$ have $b=\left(v_{i_{1}}, \ldots, v_{i_{k-1}}\right)$. Let the share of $P_{i_{k}}$ be $x \in\{0,1, \ldots, N-1\}$. Then, from eq. (9),

$$
\operatorname{Sec}(b, x)=\sum_{j=1}^{k-1} c_{j} v_{i_{j}}+c_{k} x=d_{s} \in B(=\mathcal{S}) \text {. }
$$

Define

$$
T \triangleq\{x \mid \operatorname{Sec}(b, x) \in B\} .
$$

For any fixed $b$, eq. (10) defines a bijection $\tau$ from $B$ to $T$ such that $\tau\left(d_{s}\right)=x \in T$ because $c_{k} \neq 0$. Since $d_{s}$ is uniformly distributed over $B, x$ is uniformly distributed over $T$. (Remember that $S$ is uniformly distributed over $\mathcal{S}$.) Therefore for any fixed $b$ and $b^{\prime}$,

$$
\operatorname{Pr}\left(P_{i_{k}} \text { is cheated by } b^{\prime} \mid P_{i_{1}} \cdots P_{i_{k-1}} \text { have } b\right)=\left|\tilde{\mathcal{V}}_{i_{k}}\left(b \rightarrow b^{\prime}\right)\right| /|T| \text {, }
$$

where

$$
\tilde{\mathcal{V}}_{i_{k}}\left(b \rightarrow b^{\prime}\right)=\left\{x \mid \operatorname{Sec}(b, x) \in B, \operatorname{Sec}\left(b^{\prime}, x\right) \in B \text { and } \operatorname{Sec}(b, x) \neq \operatorname{Sec}\left(b^{\prime}, x\right)\right\} .
$$

Since $\tau$ is a bijection,

$$
|T|=|B|=l \text {. }
$$

Now let's compute $\left|\tilde{\mathcal{V}}_{i_{k}}\left(b \rightarrow b^{\prime}\right)\right|$. Fix $b=\left(v_{i_{1}}, \ldots, v_{i_{k-1}}\right)$ and $b^{\prime}=\left(v_{i_{1}}^{\prime}, \ldots, v_{i_{k-1}}^{\prime}\right)$ arbitrarily. Define

$$
a \triangleq \sum_{j=1}^{k-1} c_{j} v_{i_{j}}, \quad a^{\prime} \triangleq \sum_{j=1}^{k-1} c_{j} v_{i_{j}}^{\prime}
$$


From eq. (10) and since $\tau$ is a bijection,

$$
\begin{aligned}
\left|\tilde{\mathcal{V}}_{i_{k}}\left(b \rightarrow b^{\prime}\right)\right| & =\mid\left\{x \mid a+c_{k} x \in B, a^{\prime}+c_{k} x \in B \text { and } a+c_{k} x \neq a^{\prime}+c_{k} x\right\} \mid \\
& =\mid\left\{d \mid d \in B, d-\left(a-a^{\prime}\right) \in B \text { and } a-a^{\prime} \neq 0\right\} \mid
\end{aligned}
$$

Note that $a-a^{\prime}$ is a constant for fixed $b$ and $b^{\prime}$. On the other hand, from Definition 9 , for $\forall e \neq 0$,

$$
\begin{array}{r}
\left|\left\{\left(d, d^{\prime}\right) \mid d \in B, d^{\prime} \in B, d-d^{\prime}=e\right\}\right|=1 \\
|\{d \mid d \in B, d-c \in B\}|=1
\end{array}
$$

since $d^{\prime}=d-e$. So we obtain

$$
\left|\tilde{\mathcal{V}}_{i_{k}}\left(b \rightarrow b^{\prime}\right)\right|=1
$$

for $b$ and $b^{\prime}$ such that $a-a^{\prime} \neq 0$. If $a-a^{\prime}=0$, then $\left|\tilde{V}_{i_{k}}\left(b \rightarrow b^{\prime}\right)\right|=0$ because no $d$ (or no $x$ ) satisfies $a-a^{\prime} \neq 0$. Therefore, from eq. (11),(12) and (13),

$$
\max _{b^{\prime}} \operatorname{Pr}\left(P_{i_{k}} \text { is cheated by } b^{\prime} \mid P_{i_{1}} \cdots P_{i_{k-1}} \text { have } b\right)=1 / l \text {. }
$$

Consequently, from eq. (5),

$$
P\left(\text { Cheat } \mid V_{i_{1}}, \ldots, V_{i_{k-1}}\right)=1 / l \text {. }
$$

Thus this scheme is a $(k, n, \delta)$ secure scheme such that $\delta=1 / l$. It is clear that $|\mathcal{S}|=|B|=$. Finally, from eq. (8), $\forall j,\left|\mathcal{V}_{j}\right|=N=(l-1) l+1=(|\mathcal{S}|-1) / \delta+1$. Hence, this scheme meets the equality of eq. (6).

Now the following theorem is obtained from lemma 14 and 15.

Theorem 16. If there exists a planar difference set modulo $N=l(l-1)+1$ such that $N$ is a prime, then there exists $a(k, n, \delta)$ secure secret sharing scheme which meets the equality of our bound eq. (6) such that $|\mathcal{S}|=l, \delta=1 / l, n<N$.

From proposition 10, we obtain the following corollary.

Corollary 17. Let $q$ be a prime power such that $q^{2}+q+1$ is a prime. Then, there exists a $(k, n, \delta)$ secure secret sharing scheme which meets the equality of eq. (6) such that $|\mathcal{S}|=q+1, \delta=1 /(q+1)$ and $n<q^{2}+q+1$.

Remark. Instead of publicizing a planar difference set $B$ itself, it is enough to publicize two points $\alpha^{0}$ and $\alpha^{1}$ of $P G(2,|\mathcal{S}|-1)$. According to Proposition 10, $B$ can be obtained from $\left(\alpha^{0}, \alpha^{1}\right)$. 


\subsection{Optimum scheme based on a $(N, l, \lambda)$ difference set}

Theorem 16 is generalized as follows.

Theorem 18. If there exists a $(N, l, \lambda)$ difference set $B$ in $(G F(N),+)$, then there exists $a(k, n, \delta)$ secure secret sharing scheme which meets the equality of our bound eq. (6) such that $|\mathcal{S}|=l, \delta=\lambda / l, n<N$.

The following corollary is obtained from proposition 12 .

Corollary 19. For a positive integer $t$ such that $4 t-1$ is a prime power, there exists a $(k, n, \delta)$ secure secret sharing scheme which meets the equality of our bound eq. (6) such that $|\mathcal{S}|=2 t-1, \delta=(t-1) /(2 t-1), n<4 t-1$.

\section{Tighter Bound on $\left|\mathcal{V}_{i}\right|$ under the CDV Assumption}

In this section, we use the same technique used in subseection 3.2 and, under the CDV assumption, show a lower bound on $\left|\mathcal{V}_{i}\right|$ that is more tight than proposition 5. (The CDV assumption is that $k-1$ cheaters $P_{1}, \ldots, P_{k-1}$ somehow know the secret $s$.)

In the distribution phase, suppose that cheaters $P_{i_{1}}, \ldots, P_{i_{k-1}}$ have $b=$ $\left(v_{i_{1}}, \ldots, v_{i_{k-1}}\right)$ as their shares of a secret $s$ and $P_{i_{k}}$ has $x$ as his share. That is, $\operatorname{Sec}(b, x)=s$. Fix $s$ and $b$. Let

$$
\begin{aligned}
Y^{\prime}(x) & \triangleq\left\{v_{i_{1}}^{\prime} \in \mathcal{V}_{i_{1}} \mid \operatorname{Sec}\left(v_{i_{1}}^{\prime}, v_{i_{2}}, \ldots, v_{i_{k-1}}, x\right)=s^{\prime} \in \mathcal{S}, s^{\prime} \neq s\right\} \\
W^{\prime} & \triangleq\left\{x \in \mathcal{V}_{i_{k}} \mid \operatorname{Sec}(b, x)=s\right\} .
\end{aligned}
$$

In the reconstruction phase, if $P_{i_{1}}$ opens $v_{i_{1}}^{\prime} \in Y^{\prime}(x)$, then $P_{i_{k}}$ is cheated. $W^{\prime}$ denotes the set of possible shares of $P_{i_{k}}$.

Lemma 20. For fixed $s$ and $b$ such that $\operatorname{Pr}\left(V_{i_{1}} \cdots V_{i_{k-1}}=b, S=s\right)>0$,

$$
\left|W^{\prime}\right| \geq 1 / \epsilon \text {. }
$$

Proof. Consider cheaters $P_{i_{1}}, \ldots, P_{i_{k-1}}$ such that only $P_{i_{1}}$ opens a forged share $v_{i_{1}}^{\prime}\left(\neq v_{i_{1}}\right)$. The other $P_{i_{2}}, \ldots, P_{i_{k-1}}$ open their shares honestly. The way that $P_{i_{1}}$ opens $v_{i_{1}}^{\prime}$ is as follows. First, $P_{i_{1}}$ chooses $\hat{x} \in W^{\prime}$ such that

$$
\operatorname{Pr}\left(V_{i_{k}}=\hat{x} \mid V_{i_{1}} \cdots V_{i_{k-1}}=b, S=s\right)=\max _{x \in W} \operatorname{Pr}\left(V_{i_{k}}=x \mid V_{i_{1}} \cdots V_{i_{k-1}}=b, S=s\right) .
$$

Then, $P_{i_{1}}$ opens $v_{i_{1}}^{\prime} \in Y^{\prime}(\hat{x})$ arbitrarily. In this case, $P_{i_{k}}$ is cheated if his share is $\hat{x}$. For these specific cheaters, in eq. (4),

$$
\begin{aligned}
& \max _{b^{\prime}} \operatorname{Pr}\left(P_{i_{k}} \text { is cheated by } b^{\prime} \mid P_{i_{1}} \cdots P_{i_{k-1}} \text { have } b \text {. They also know } s\right) \\
\geq & \operatorname{Pr}\left(P_{i_{k}} \text { is cheated by } v_{i_{1}}^{\prime} \mid P_{i_{1}} \cdots P_{i_{k-1}} \text { have } b . \text { They also know } s\right) \\
\geq & \operatorname{Pr}\left(V_{i_{k}}=\hat{x} \mid V_{i_{1}} \cdots V_{i_{k-1}}=b, S=s\right)
\end{aligned}
$$




$$
\begin{aligned}
& =\max _{x \in W^{\prime}} \operatorname{Pr}\left(V_{i_{k}}=x \mid V_{i_{1}} \cdots V_{i_{k-1}}=b, S=s\right) \\
& \geq\left|W^{\prime}\right|^{-1} \sum_{x \in W^{\prime}} \operatorname{Pr}\left(V_{i_{k}}=x \mid V_{i_{1}} \cdots V_{i_{k-1}}=b, S=s\right) \\
& \geq\left|W^{\prime}\right|^{-1}
\end{aligned}
$$

Since the scheme is $\epsilon$-robust, $\epsilon \geq E\left[\left|W^{\prime}\right|^{-1}\right]=\left|W^{\prime}\right|^{-1}$.

Therefore, we obtain eq. (14).

Lemma 21. For $\forall x \in W^{\prime},\left|Y^{\prime}(x)\right| \geq(|\mathcal{S}|-1) / \epsilon$.

Proof. From lemma 20, $\left|\left\{y \in \mathcal{V}_{i_{1}} \mid \operatorname{Sec}\left(y, v_{2}, \ldots, v_{k-1}, x\right)=s^{\prime}\right\}\right| \geq 1 / \epsilon$.

Therefore,

$$
\begin{aligned}
\left|Y^{\prime}(x)\right| & =\left|\bigcup_{s^{\prime} \in \mathcal{S}, s^{\prime} \neq s}\left\{y \in V_{i_{1}} \mid \operatorname{Sec}\left(v_{i_{1}}^{\prime}, v_{i_{2}}, \ldots, v_{i_{k-1}}, x\right)=s^{\prime}\right\}\right| \\
& =\sum_{s^{\prime} \in \mathcal{S}, s^{\prime} \neq s}\left|\left\{y \in \mathcal{V}_{i_{1}} \mid \operatorname{Sec}\left(y, v_{2}, \ldots, v_{k-1}, x\right)=s^{\prime}\right\}\right| \\
& \geq \sum_{s^{\prime} \in \mathcal{S}, s^{\prime} \neq s} 1 / \epsilon \\
& =(|\mathcal{S}|-1) / \epsilon .
\end{aligned}
$$

Now, our lower bound on $\left|\mathcal{V}_{i}\right|$ is as follows.

Theorem 22. In a $(k, n, c)$ robust secret sharing scheme,

$$
\left|\mathcal{V}_{i}\right| \geq \frac{|\mathcal{S}|-1}{\epsilon^{2}}+1
$$

Proof. Consider a probabilistic $P_{i_{1}}$ such as shown in the proof of Theorem 8 . For such $P_{i_{1}}$, let's compute

$$
E\left[\operatorname{Pr}\left(P_{i_{k}} \text { is cheated by } v_{i_{1}}^{\prime} \mid P_{i_{1}} \ldots P_{i_{k-1}} \text { have } b \text { and they know } s\right],\right.
$$

where $E$ is taken over $v_{i_{1}}^{\prime}$ and $\operatorname{Pr}()$ is taken over $x \in W^{\prime}$. Then from lemma 21,

$E_{v_{i_{1}}^{\prime}}\left[\operatorname{Pr}_{x \in W^{\prime}}\left(P_{i_{k}}\right.\right.$ is cheated by $v_{i_{1}}^{\prime} \mid P_{i_{1}} \cdots P_{i_{k-1}}$ have $b$ and they know $\left.s\right]$

$$
=E_{x \in W^{\prime}}\left[\underset{v_{i_{1}}^{\prime}}{\operatorname{Pr}}\left(P_{i_{k}} \text { is cheated by } v_{i_{1}}^{\prime} \mid P_{i_{1}} \ldots P_{i_{k-1}} \text { have } b \text { and they know } s\right]\right.
$$$$
=E_{x \in W^{\prime}}\left[\left|Y^{\prime}(x)\right| /\left(\left|\mathcal{V}_{i_{1}}\right|-1\right)\right]
$$$$
\geq(|\mathcal{S}|-1) / \epsilon\left(\left|\mathcal{V}_{i_{1}}\right|-1\right) \text {. }
$$

Therefore

$$
\begin{aligned}
& \max _{v_{i_{1}}^{\prime}} \operatorname{Pr}\left(P_{i_{k}} \text { is cheated by } v_{i_{1}}^{\prime} \mid P_{i_{1}} \cdots P_{i_{k-1}} \text { have } b \text { and they know } s\right) \\
\geq & E_{v_{i_{1}}^{\prime}}\left[\operatorname{Pr}\left(P_{i_{k}} \text { is cheated by } v_{i_{1}}^{\prime} \mid P_{i_{1}} \cdots P_{i_{k-1}} \text { have } b \text { and they know } s\right)\right] \\
\geq & (|\mathcal{S}|-1) / \epsilon\left(\left|V_{i_{1}}\right|-1\right) .
\end{aligned}
$$


Hence

$$
\begin{gathered}
\max _{b^{\prime}} \operatorname{Pr}\left(P_{i_{k}} \text { is cheated by } b^{\prime} \mid P_{i_{1}} \cdots P_{i_{k-1}} \text { have } b \text { and they know } s\right) \\
\geq(|\mathcal{S}|-1) / \epsilon\left(\left|V_{i_{1}}\right|-1\right) .
\end{gathered}
$$

Consequently, in a $(k, n, \epsilon)$ robust secret sharing scheme,

$$
\begin{aligned}
\varepsilon & \geq E\left[\max _{b^{\prime}} \operatorname{Pr}\left(P_{i_{k}} \text { is cheated by } b^{\prime} \mid P_{i_{1}} \cdots P_{i_{k-1}} \text { have } b \text {. They also know } s\right)\right] \\
& \geq(|\mathcal{S}|-1) / \epsilon\left(\left|\mathcal{V}_{i_{1}}\right|-1\right) .
\end{aligned}
$$

Then, eq. (15) is obtained.

\section{References}

1. M. Tompa and H. Woll. "How to share a secret with cheaters". In Journal of Cryptology, vol.1, pages 133-138, 1988.

2. A. Shamir. "How to Share a Secret". In Communications of the ACM, vol.22, no.11, pages 612-613, 1979.

3. G.R. Blakely, "Safeguarding cryptographic keys". In Proc. of the AFIPS 1979 National Computer Conference, vol.48, pages 313-317, 1979.

4. E.D. Karnin, J.W.Green, and M.E. Hellman. "On secret sharing systems". In IEEE Trans. IT-29, No.1, pages 35-41, 1982.

5. M. Carpentieri, A. De Santis, and U. Vaccaro. "Size of Shares and Probability of Cheating in Threshold Schernes". In Proc. of Eurocrypto'93, Lecture Notes in Computer Science, LNCS 765, Springer Verlag, pages 118-125, 1993.

6. F.J. MacWilliams and N.J.A. Sloane. "The theory of error-correcting codes". In North-Holland, pages 397-398, 1981.

7. T.Beth,T, D.Jungnickel and H.Lenz. "Design Theory". In Cambridge University Press, pages 260-264, 1993.

8. R.J. McEliece and D.V. Sarwate. "On sharing secrets and Reed-Solomon codes". In Comm.ACM, 24, pages 583-584, 1981.

9. T. Rabin and M. Ben-Or. "Verifiable secret sharing and multiparty protocols with honest majority". In Proc. 21st ACM Symposium on Theory of Computing, pages $73-85,1989$.

10. E.F. Brickell and D.R. Stinson. "The Detection of Cheaters in Threshold Schemes". In SIAM J. DISC. MATH, Vol.4, No.4, pages 502-510, 1991.

11. M. Carpentieri. "A perfect threshold secrety sahring scheme to identify cheaters". In Designs, Codes and Cryptography, vol.5, no.3, pages 183-187, 1995.

12. K. Kurosawa, S. Obana, and W. Ogata. "t-cheater identifiable $(k, n)$ threshold secret sharing schemes". In Proc. of Crypt'95, Lecture Notes in Computer Science, LNCS 963, Springer Verlag, pages 410-423, 1995.

13. D.R. Stinson. "Cryptography: Theory and Practice". In CRC Press, pages 330$331,1995$. 